\title{
Towards Building Intrusion Detection Systems for Multivariate Time-Series Data
}

\author{
ChangMin Seong ${ }^{1}$, YoungRok Song ${ }^{2}$, Jiwung Hyun ${ }^{2}$, and Yun-Gyung Cheong ${ }^{2(\otimes)}$ \\ 1 Department of Computer Software, Sungkyunkwan University, Suwon, South Korea \\ 2 Department of Artificial Intelligence, Sungkyunkwan University, Suwon, South Korea \\ ygcheong@gmail.com
}

\begin{abstract}
Recent network intrusion detection systems have employed machine learning and deep learning algorithms to defend against dynamically evolving network attacks. While most previous studies have focused on detecting attacks which can be determined based on a single time instant, few studies have paid attention to subsequence outliers, which require inspecting consecutive points in time for detection. To address this issue, this paper applies a time-series anomaly detection method in an unsupervised learning manner. To this end, we converted the UNSW-NB15 dataset into the time-series data. We carried out a preliminary evaluation to test the performance of the anomaly detection on the created timeseries network dataset as well as on a time-series dataset obtained from sensors. We analyze and discuss the results.
\end{abstract}

Keywords: Time series · Intrusion detection system · Stacked RNN · Unsupervised learning · Anomaly detection

\section{Introduction}

Due to the rapid development and popularization of networks, security issues are also becoming an important issue. In order to solve these security issues, a network intrusion detection system (NIDS) has been widely used. A NIDS is a system that reads network packets and detects attack traffic and is known as an effective defense method against network security issues. During the last decade, network security systems have been developed by employing various time-series intrusion detection techniques. Pankaj et al. [21] propose a Long Short Term Memory Networks based Encoder-Decoder scheme for Anomaly Detection (EncDec-AD) that learns to reconstruct normal time-series behavior. Kyle et al. [22] demonstrate the effectiveness of LSTM and propose dynamic thresholding approach using LSTMs. Ding et al. [23] propose a real-time anomaly detection algorithm (RADM) based on Hierarchical Temporal Memory (HTM) and Bayesian Network (BN). Park et al. [24] introduced a long short-term memory-based variational autoencoder (LSTM-VAE) that fuses signals and reconstructs expected distribution.

Furthermore, unsupervised learning algorithms have been getting more attention owing to their advantage of training the models without labels during the training phase $[11,12]$. In the unsupervised methods, attacks are generally detected by regarding them as outliers or anomalies. More details about outlier detection can be found in $[1,2,10]$. 
Time-series data mean the data annotated with time stamps, collected at regular time intervals. Depending on what is considered an outlier, time-series outliers are largely divided into two types: point outliers and subsequence outliers [2]. A point outlier means an outlier of which value is significantly different from the values of the surrounding data in the overall flow of data in time order as shown in Fig. 1. In the figure, a point between 10 and 11 can be regarded as normal with a global perspective where similar data values exist between 21 and 22, but it is determined as an outlier considering the values of its neighbors with a local perspective [3]. These outliers can be determined relying on their characteristics at a specific time instant.

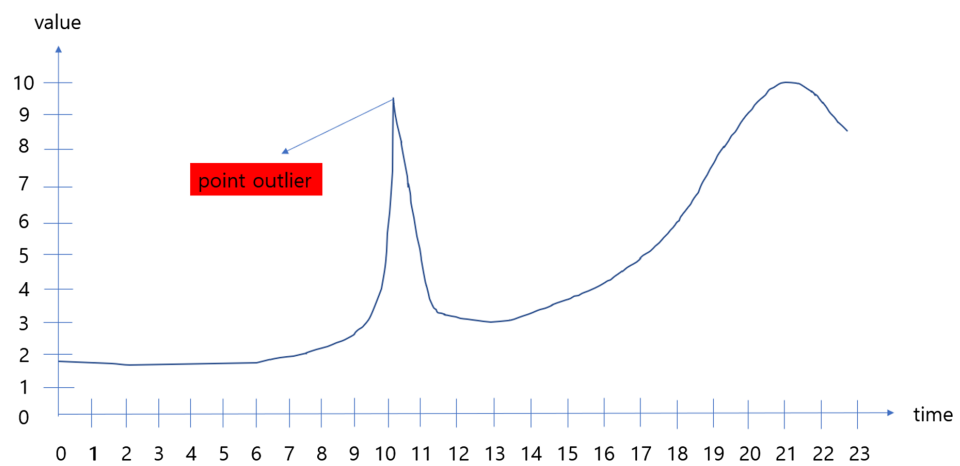

Fig. 1. An illustration of a point outlier where samples between 10 and 11 are spiking, distinguished from their neighboring data.

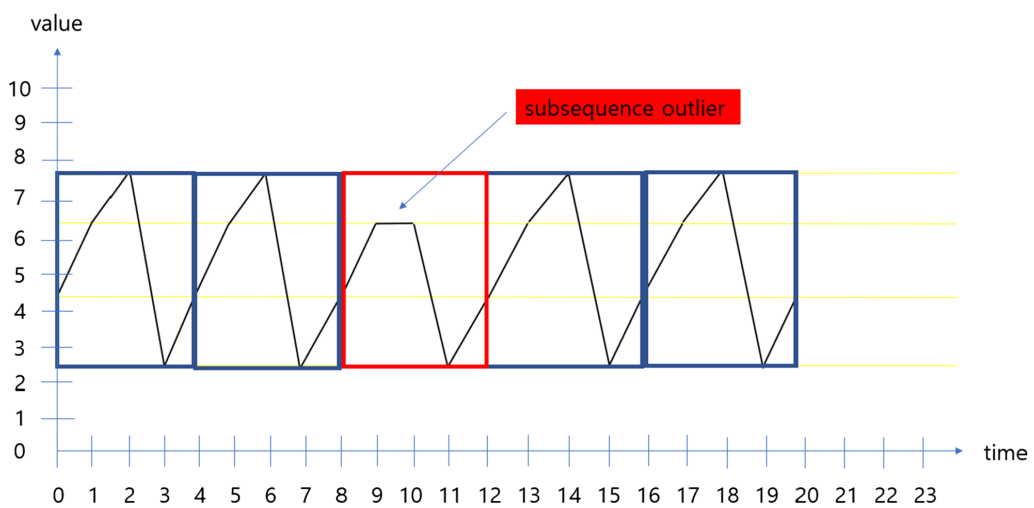

Fig. 2. An illustration of a subsequence outlier which is represented in the red box. The data values are within the minimum and the maximum of normal data, and yet the overall pattern is different from the rest. (Color figure online)

On the contrary, a subsequence outlier can be found only by inspecting consecutive instants in time. A subsequence outlier shows a pattern that deviates from the normal repetitive patents as shown in Fig. 2. The points between 9 and 10 can be regarded as normal when simply looking at the numerical values, but it is determined as an outlier 


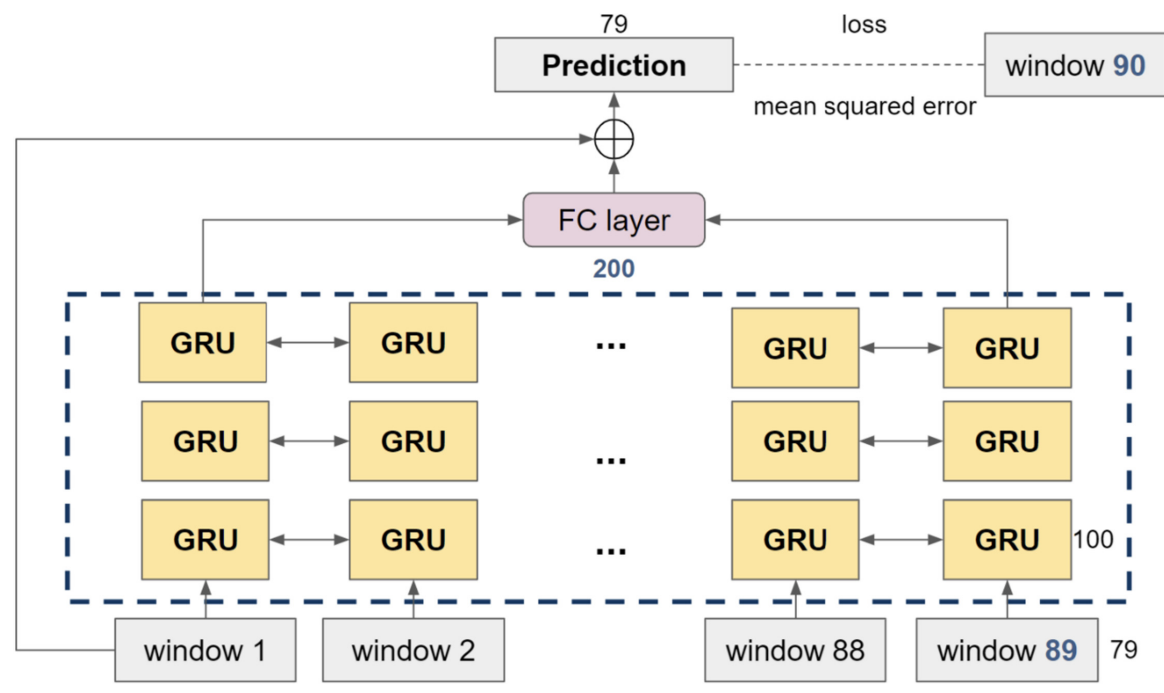

Fig. 3. The model structure uses stacked RNN(GRU) models. For the sliding window, which is the time interval the model trains the specific pattern, set to 90 . Using the output of previous 89 data, the model predicts 90 th data in the window. The numbers 79 denote the number of features excluding the time feature, 100 denotes the number of hidden cells of GRU, and 200 denotes the number of nodes of the FC (Fully Connected) layer.

since its pattern deviated from the repeating patterns between 1 and 2,5 and 6, 13 and 14, and 17 and 18 [3]. Therefore, it is necessary to detect both outliers for building an intrusion detection system for practical domains. However, most previous studies have focused on detecting point outliers $[6,20]$.

To address this issue, this paper attempts to detect attacks using multivariate timeseries network data. Since time-series network datasets are rarely available, we created a time-series network dataset using the UNSW-NB15 network dataset [7, 13-16]. As an experimental model, we employ an unsupervised approach which contains a stacked RNN model, as was provided by the DACON's HAICon2021 competition [17]. The approach showed a good performance, achieving F1 of 0.926 when the provided code was run on the HAI 2.0 dataset [4]. We carried out preliminary evaluations to test if this approach can be applied to the time-series network data.

\section{Model}

We use a stacked RNN (GRU) model [5] for learning time-series data in an unsupervised learning manner to detect attacks, which was provided as the baseline model for the HAICon2021 competition. This model uses a three-layer bidirectional GRU with 100 hidden cells as illustrated in Fig. 3. We use the experiment configuration that was set for the baseline model for comparison in the future research. We train the model for 32 epochs keeping the best model parameters, and the parameters that result in the best loss were chosen for evaluation. The window size was set as 90 . 


\section{Time-Series Anomaly Detection Datasets}

To evaluate the time-series anomaly detection system we selected two datasets, UNSWNB15 dataset [7] and HAI 2.0 dataset [4]. The UNSW-NB15 dataset is converted into a time-series format.

\subsection{The UNSW-NB15 Dataset}

The UNSW-NB15 dataset is widely used for benchmarking network intrusion detection systems. The dataset contains 9 network attack behaviors which are Fuzzers, Analysis, Backdoors, DoS, Exploits, Generic, Reconnaissance, Shellcode, and Worms. The data are provided in two formats, raw traffic packet file and CSV file containing features extracted from captured network flows. We follow Ge et al. [8] to convert the packet data into a time-series format.

Feature Extraction: The raw traffic packets from the UNSW-NB15 dataset were captured using the IXIA PerfectStorm tool and are provided in the PCAP file format [7]. We first select and extract packet fields from the PCAP file using the TShark analyzer tool. Details of the selected fields are shown in Table 1.

Table 1. Detailed information of extracted fields from network packets.

\begin{tabular}{l|l}
\hline Feature & Field detail \\
\hline frame & frame.time_epoch, frame.len \\
\hline ip & ip.src, ip.dst, ip.ttl \\
\hline tcp & tcp.srcport, tcp.dstport, tcp.stream, tcp.len, tcp.checksum \\
\hline udp & udp.srcport, udp.dstport, udp.stream, udp.checksum, udp.length \\
\hline
\end{tabular}

The UNSW-NB15 CSV file contains the flow-based features of labeled flow data. The description of 49 features in the file are listed in Table 2. Each flow is labelled as 0 for normal records and 1 for attacks.

Packet Labelling: After extracting the features from each packet, we sort them in the chronological order using the frame.time_epoch feature, which indicates the time information of the packet. The packets in the PCAP file are labelled using the labels in the CSV file. It has information about packets transmitted and a label denoting normal or attack. A label can be created by using the label feature value of the flow which contains the packet.

The process of determining whether a particular packet belongs to a flow is as follows. First, frame.time_epoch of the PCAP file is matched with the Stime value (the 29th field) and the Ltime value (the 30th field) of the CSV file. Among the data matched with the packet, we extracted the data that matches the ip.src and ip.dst of the PCAP with the 
Table 2. Description of features.

\begin{tabular}{l|l|l|l|l|l}
\hline Number & Description & Number & Description & Number & Description \\
\hline 1 & srcip & 18 & Dpkts & 35 & ackdat \\
\hline 2 & sport & 19 & swin & 36 & is_sm_ips_ports \\
\hline 3 & dstip & 20 & dwin & 37 & ct_state_ttl \\
\hline 4 & dsport & 21 & stcpb & 38 & ct_flw_http_mthd \\
\hline 5 & proto & 22 & dtcpb & 39 & is_ftp_login \\
\hline 6 & state & 23 & smeansz & 40 & ct_ftp_cmd \\
\hline 7 & dur & 24 & dmeansz & 41 & ct_srv_src \\
\hline 8 & sbytes & 25 & trans_depth & 42 & ct_srv_dst \\
\hline 9 & dbytes & 26 & res_bdy_len & 43 & ct_dst_ltm \\
\hline 10 & sttl & 27 & Sjit & 44 & ct_src_ltm \\
\hline 11 & dttl & 28 & Djit & 45 & ct_src_dport_ltm \\
\hline 12 & sloss & 29 & Stime & 46 & ct_dst_sport_ltm \\
\hline 13 & dloss & 30 & Ltime & 47 & ct_dst_src_ltm \\
\hline 14 & service & 31 & Sintpkt & 48 & attack_cat \\
\hline 15 & Sload & 32 & Dintpkt & 49 & Label \\
\hline 16 & Dload & 33 & tcprtt & & \\
\hline 17 & Spkts & 34 & synack & & \\
\hline
\end{tabular}

first field srcip and the third field dstip of the CSV file. Finally, for TCP, we matched tcp.srcport and tcp.dstport in the PCAP file, and in the case of UDP, udp.srcport and udp.dstport in the PCAP file with the 2nd field sport, and 4th field dsport of the CSV file, and the label of the matched file becomes the label of the corresponding PCAP file. If there is no matching data, it is infeasible to determine whether it is normal or an attack, hence, we removed the corresponding packet. $T c p$ information and $u d p$ information are integrated into one common information, and then in the case of ip.src and ip.dst, they are used up to map the PCAP file and the CSV information and then removed. Finally, in the created time-series network data, there are 9 features: frame.time_epoch, frame.len, ip.ttl, srcport, dstport, stream, checksum, len, and label. We removed the label from the data for train, validation and test, since we apply unsupervised learning to dataset, we only used the label for evaluation for validation and test. In total, there are 295,342 time-series data with 277,828 normal data and 17,514 attack data.

Preprocessing: For the source port and destination port features, the port numbers greater than 49,152 are labelled as 2, the numbers greater than 1,024 are labelled to 1 , and the numbers lower than 1,024 are labelled to 0 since they are divided to dynamic port, registered port and well-known port. Then numerical features were scaled to fit 0 to 1 using a min-max scaler. 


\subsection{The HAI 2.0 Dataset}

The HAI 2.0 dataset is a time-series dataset created for attack detection in cyber-physical systems such as railways, water-treatment, and power plants [4]. The data were collected from the four processes: the boiler process, the turbine process, the water-treatment process, and the HIL simulation. Data samples were collected every second and consist of 80 features. Normal data were collected for 7 continuous days, and the attack data include 38 different attack types. The data are sorted in the increasing order of time feature in the format of "yyyy-MM-dd hh:mm:ss.". Other features contain information associated with the processes such as temperature setpoint, water level setpoint and motor speed.

Preprocessing: To preprocess the data, the timestamp features were dropped, and the numerical features were scaled with a min-max scaler similar to UNSW-NB15 [17]. For some features, of which maximum value and minimum value are the same, we set these features as 0 . After scaling features, we applied an exponential weighted function in python function "ewm" with 0.9 for alpha for noise smoothing.

\section{Experiments}

We compare and analyze the anomaly detection system performance using the UNSWNB15 and the HAI 2.0 dataset. We convert attack detection into an anomaly detection problem by assuming the attack to be anomalous.

\subsection{Data Preparation}

For both datasets, an unsupervised learning was conducted to train the model using only normal data. We divided the time-series network dataset into training, validation, and test datasets in a ratio of $8: 1: 1$. Then, since the attack data is also included in the training datasets for the time-series network data, we removed attack data in the training datasets. The number of instances for each dataset is presented in Table 3.

Table 3. Simple statistics of processed UNSW-NB15 dataset.

\begin{tabular}{l|r|l|l}
\hline & Training & Validation & Test \\
\hline Normal & 226,240 & 25,706 & 25,882 \\
\hline Attack & 0 & 3,828 & 3,652 \\
\hline Total & 226,240 & 29,534 & 29,534 \\
\hline
\end{tabular}

However, there are no labels in the test dataset of HAI 2.0 dataset. For the evaluation, we divided the validation dataset, which has labels, into the validation dataset (first 50\%) and the test dataset (last 50\%). Table 4 shows the simple statistics of the processed dataset. 
Table 4. Simple statistics of the processed HAI 2.0 dataset.

\begin{tabular}{l|r|l|l}
\hline & Training & Validation & Test \\
\hline Normal & 965,603 & 21,060 & 21,512 \\
\hline Attack & 0 & 540 & 89 \\
\hline Total & 965,603 & 21,600 & 21,601 \\
\hline
\end{tabular}

\subsection{Training}

As described in Fig. 3, the model is trained to predict the last sample in the given time window when the preceding samples are given. In order to predict whether the last sample is an anomaly the model is only trained with windows containing normal samples. Theoretically the model will predict the last sample as close as possible to the normal sample given the preceding sample. Therefore, if the difference between the prediction and true last sample is significant, we consider the last sample to be an anomaly. We predict the last sample of the window as an anomaly if the difference is greater than a predetermined threshold. The parameters for training the model are provided in Table 5. The stride means how much data to skip during training.

Table 5. Model parameters and configurations.

\begin{tabular}{l|l|l|l}
\hline Parameter & Value/Name & Parameter & Value/Name \\
\hline n_hidden & 100 & n_layers & 3 \\
\hline batch_size & 512 & num_epochs & 32 \\
\hline window_size & 90 & stride & 10 \\
\hline loss & MSE & optimizer & AdamW \\
\hline scheduler & $\mathrm{X}$ & dropout & $\mathrm{X}$ \\
\hline
\end{tabular}

\subsection{The Evaluation Metrics}

There are various evaluation metrics such as precision, recall, and $F 1$ that are frequently used. However, the evaluation metric of time-series data needs to consider various factors such as the diversity of detected attacks and the accuracy of detection as illustrated in Fig. 4.

For example, as shown in Fig. 4, Model 2 detects 3 anomaly instances between 0 and 3, and Model 1 detects 2 instances, one between 1 and 2 and the other between 6 and 7. In terms of accuracy, Model 2 outperforms Model 1. However, considering that Model 2 does not detect anomalies between 6 and 8 time slots, it is hard to determine which model performs better. TaPR [19] is an evaluation metric that considers these factors. TaP, which corresponds to precision, is an evaluation metric indicating whether 

A
Anomaly

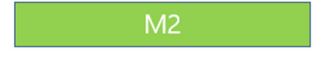

M1

\section{M1}

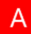

A

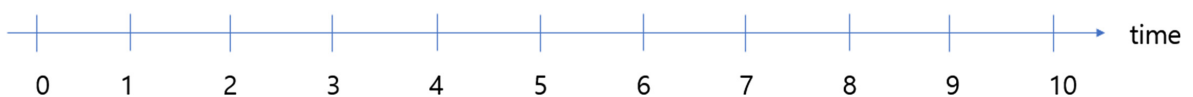

Fig. 4. Illustration of time-series anomaly detection where the two different models Model 1 and Model 2 are used, modified from [18]. The $\mathrm{X}$-axis indicates time, and $A$ indicates the time slots where an anomaly exists. $M 1$ indicates the anomalies that Model 1 detects, and $M 2$ indicates the anomalies that Model 2 detects.

the prediction finds outliers with less false positives. TaR, which corresponds to recall, is an evaluation metric indicating the diversity of the anomalies. Using the detection score $\mathrm{TaP}^{\mathrm{d}}$ (resp. $\mathrm{TaR}^{\mathrm{d}}$ ) and the portion score $\mathrm{TaP}^{\mathrm{p}}$ (resp. TaR ${ }^{\mathrm{p}}$ ), TaP and TaR can be calculated as follows:

$$
\begin{gathered}
\mathrm{TaP}=\alpha \times \mathrm{TaP}^{\mathrm{d}}+(1-\alpha) \times \mathrm{TaP}^{\mathrm{p}} \\
\mathrm{TaR}=\alpha \times \mathrm{TaR}^{\mathrm{d}}+(1-\alpha) \times \mathrm{TaR}^{\mathrm{p}}
\end{gathered}
$$

where $\alpha$ controls the ratio of $\mathrm{TaP}^{\mathrm{d}}\left(\right.$ resp. $\left.\mathrm{TaR}^{\mathrm{d}}\right)$ and $\mathrm{TaP}^{\mathrm{p}}\left(\right.$ resp. TaR ${ }^{\mathrm{p}}$ ), and its value is between 0 and 1 [9].

\section{The Experiment Results}

This section reports the evaluation results. The figures below show the error and attack distribution of the time-series network data created in this paper and the HAI 2.0 data, respectively (Figs. 5 and 6).

Using the experimental results of validation data, the threshold was set to 0.04 for the HAI 2.0 data, and the threshold was set to 0.2 for time-series network data. The two dataset show different properties. In the HAI 2.0 data, the attack data tends to be greater than the normal data, while in the time-series network data values are relatively evenly distributed. In addition, in the case of the HAI 2.0 dataset, the number of normal data is overwhelmingly larger than that of attack data, unlike the time-series network data. As the evaluation metric, we use TaPR described in Sect. 4.3. The analyses of the results are shown in the following Tables 6 and 7. 

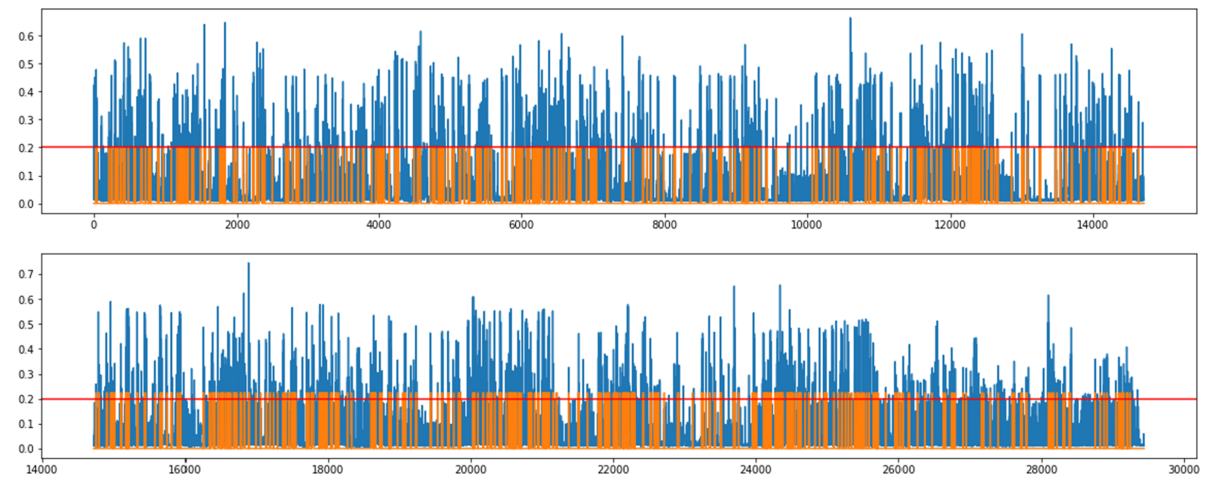

Fig. 5. Distribution of error and attack in validation dataset of the time-series network dataset. The $\mathrm{x}$-axis indicates the order of the data, and the $\mathrm{y}$-axis indicates the absolute difference of (answer - guess). The orange line indicates the attack position, and the blue line indicates the size of the error. The red line is the threshold value that separates the boundary between normal and attack. (Color figure online)
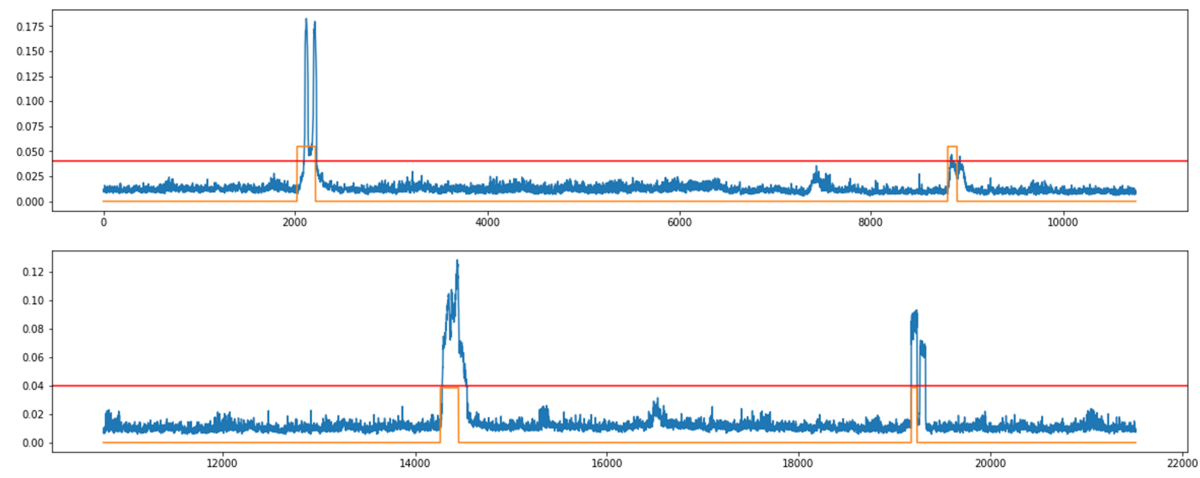

Fig. 6. Distribution of error and attack in validation dataset of the HAI 2.0 dataset. The $x$-axis indicates the order of the data, and the y-axis indicates the absolute difference of (answer - guess). The orange line indicates the attack position, and the blue line indicates the size of the error. The red line is the threshold value that separates the boundary between normal and attack. (Color figure online)

Table 6. Detection performance results of UNSW-NB15 data.

\begin{tabular}{l|l}
\hline Evaluation metric & UNSW-NB15 data \\
\hline F1 & 0.737 \\
\hline TaP & 0.731 \\
\hline TaR & 0.743 \\
\hline
\end{tabular}


Table 7. Detection performance results of HAI 2.0 data.

\begin{tabular}{l|l}
\hline Evaluation metric & HAI 2.0 data \\
\hline F1 & 0.926 \\
\hline $\mathrm{TaP}$ & 0.861 \\
\hline $\mathrm{TaR}$ & 1.000 \\
\hline
\end{tabular}

The F1 scores are 0.926 for HAI 2.0 data and 0.737 for time-series network data. The TaP and TaR scores are 0.861 and 1.000 for HAI 2.0 data, and 0.731 and 0.743 for the time-series network data, respectively. This indicates that the model performs better with the HAI 2.0 dataset which contains sensor data.

There are two main factors that account for the poor performance of the time-series network dataset. First, the number of features in the time-series network dataset may be insufficient. In the case of the HAI 2.0 dataset, there are about 80 features, in the case of the time-series network data, only about 10 features were used, making it difficult to determine its anomaly. The other reason is that time-series network data are not complete time-series. In the case of HAI 2.0 dataset, data is generated every second, but in the case of the time-series network dataset, since packets are not transmitted at a specific period, it is difficult to generate data at regular intervals. Moreover, since the attack data is removed from the training data of the time-series network data to learn the normal data only, the time information becomes more irregular.

\section{Conclusion}

While unsupervised deep learning models have shown great performances in detecting attacks that are point outliers, little has been researched on detecting subsequence outliers. For building a NIDS which can detect subsequence outliers, we first created the time-series network data by processing the UNSW-NB15 dataset. We carried out preliminary experiments using both the HAI 2.0 dataset and the time-series network dataset we created, using a stacked RNN model in an unsupervised manner. The results show that the model performs better with run on the HAI 2.0 dataset than tested on the time-series network dataset. The model achieved F1 scores of 0.926 for the HAI 2.0 data and 0.737 for the time-series network data. The TaP and TaR scores are 0.861 and 1.000 for the HAI 2.0 data, and 0.731 and 0.743 for the time-series network data. The lack of data and insufficient features of the time-series network data can account for its poor performance. We expect that more studies on time-series network data attack detection in the future will help solve these shortcomings.

Acknowledgement. This work was supported by Institute of Information \& communications Technology Planning \& Evaluation (IITP) grant funded by the Korea government (MSIT) (No. 2020-0-00952, Development of 5G Edge Security Technology for Ensuring 5G+ Service Stability and Availability). 


\section{References}

1. Braei, M., Wagner, S.: Anomaly detection in univariate time-series: a survey on the state-ofthe-art. ArXiv abs/2004.00433 (2020)

2. Bl'azquez-Garc'ia, A., et al.: A review on outlier/anomaly detection in time series data. ACM Comput. Surv. (CSUR) 54, 1-33 (2021)

3. “Anomaly Detection in Time Series: 2021”, neptune.ai. 19 July 2021. https://neptune.ai/blog/ anomaly-detection-in-time-series. Accessed 5 Sept 2021

4. Shin, H.-K., Lee, W., Yun, J.-H., Kim, H.: HAI 1.0: HIL-based augmented ICS security dataset. In: 13th USENIX Workshop on Cyber Security Experimentation and Test (2020)

5. Cho, K., et al.: Learning phrase representations using RNN encoder-decoder for statistical machine translation. In: EMNLP (2014)

6. Sandosh, S., Govindasamy, V., Akila, G.: Enhanced intrusion detection system via agent clustering and classification based on outlier detection. Peer-to-Peer Network. Appl. 13(3), 1038-1045 (2020). https://doi.org/10.1007/s12083-019-00822-3

7. Moustafa, N., Slay, J.:UNSW-NB15: a comprehensive data set for network intrusion detection systems (UNSW-NB15 network data set). In: Military Communications and Information Systems Conference (MilCIS). IEEE (2015)

8. Ge, M., et al.: Deep learning-based intrusion detection for IoT networks. In: 2019 IEEE 24th Pacific Rim International Symposium on Dependable Computing (PRDC), pp. 256-25609 (2019)

9. Hwang, W.-S., Yun, J.-H., Kim, J., Kim, H.: Time-series aware precision and recall for anomaly detection: considering variety of detection result and addressing ambiguous labeling, pp. 2241-2244 (2019). https://doi.org/10.1145/3357384.3358118

10. Gupta, M., Gao, J., Aggarwal, C.C., Han, J.: Outlier detection for temporal data: a survey. IEEE Trans. Knowl. Data Eng. 26(9), 2250-2267 (2014). https://doi.org/10.1109/TKDE.201 3.184

11. Song, Y., Hyun, S., Cheong, Y.-G.: A systematic approach to building autoencoders for intrusion detection. In: Park, Y., Jadav, D., Austin, T. (eds.) SVCC 2020. CCIS, vol. 1383, pp. 188-204. Springer, Cham (2021). https://doi.org/10.1007/978-3-030-72725-3_14

12. Song, Y., Hyun, S., Cheong, Y.-G.: Analysis of autoencoders for network intrusion detection. Sensors 21(13), 4294 (2021). https://doi.org/10.3390/s21134294

13. Moustafa, N., Slay, J.: The evaluation of network anomaly detection systems: statistical analysis of the UNSW-NB15 dataset and the comparison with the KDD99 dataset. Inf. Secur. J. Glob. Perspect., 1-14 (2016)

14. Moustafa, N., et al.: Novel geometric area analysis technique for anomaly detection using trapezoidal area estimation on large-scale networks. IEEE Trans. Big Data (2017)

15. Moustafa, N., Creech, G., Slay, J.: Big data analytics for intrusion detection system: statistical decision-making using finite dirichlet mixture models. In: Palomares Carrascosa, I., Kalutarage, H. K., Huang, Y. (eds.) Data Analytics and Decision Support for Cybersecurity. DA, pp. 127-156. Springer, Cham (2017). https://doi.org/10.1007/978-3-319-59439-2_5

16. Sarhan, M., Layeghy, S., Moustafa, N., Portmann, M.: NetFlow datasets for machine learningbased network intrusion detection systems. In: Deze, Z., Huang, H., Hou, R., Rho, S., Chilamkurti, N. (eds.) BDTA/WiCON -2020. LNICSSITE, vol. 371, pp. 117-135. Springer, Cham (2021). https://doi.org/10.1007/978-3-030-72802-1_9

17. "HAI DataSet Baseline Model", DACON, 2 August 2021. https://dacon.io/competitions/off icial/235757/codeshare/3009?page=1\&dtype=recent. Accessed 5 Sept 2021

18. "[Paper Review] Evaluation Metrics for Time Series Anomaly Detection”, DSBA, 23 September 2020. http://dsba.korea.ac.kr/seminar/?pageid=3\&mod=document\&uid=1332. Accessed 6 Sept 2021 
19. Hwang, W.-s., Yun, J.-H., Kim, J., Kim, H.: Time-series aware precision and recall for anomaly detection - considering variety of detection result and addressing ambiguous labeling. In: CIKM 2019: Proceedings of the 28th ACM International Conference on Information and Knowledge Management (2019)

20. Devan, P., Khare, N.: An efficient XGBoost-DNN-based classification model for network intrusion detection system. Neural Comput. Appl. 32(16), 12499-12514 (2020). https://doi. org/10.1007/s00521-020-04708-x

21. Malhotra, P., et al.: LSTM-based encoder-decoder for multi-sensor anomaly detection. arXiv preprint arXiv:1607.00148 (2016)

22. Hundman, K., et al.: Detecting spacecraft anomalies using lstms and nonparametric dynamic thresholding. In: Proceedings of the 24th ACM SIGKDD International Conference on Knowledge Discovery \& Data Mining (2018)

23. Ding, N., et al.: Multivariate-time-series-driven real-time anomaly detection based on bayesian network. Sensors 18(10), 3367 (2018)

24. Park, D., Hoshi, Y., Kemp, C.C.: A multimodal anomaly detector for robot-assisted feeding using an lstm-based variational autoencoder. IEEE Rob. Autom. Lett. 3(3), 1544-1551 (2018)

Open Access This chapter is licensed under the terms of the Creative Commons Attribution 4.0 International License (http://creativecommons.org/licenses/by/4.0/), which permits use, sharing, adaptation, distribution and reproduction in any medium or format, as long as you give appropriate credit to the original author(s) and the source, provide a link to the Creative Commons license and indicate if changes were made.

The images or other third party material in this chapter are included in the chapter's Creative Commons license, unless indicated otherwise in a credit line to the material. If material is not included in the chapter's Creative Commons license and your intended use is not permitted by statutory regulation or exceeds the permitted use, you will need to obtain permission directly from the copyright holder. 